\title{
ON A NONCLASSICAL PROBLEM FOR THE HEAT EQUATION AND THE FELLER SEMIGROUP GENERATED BY IT
}

\author{
KOPYTKO B.I. ${ }^{1}$, NOVOSYADLO A.F. ${ }^{2}$
}

\begin{abstract}
The initial boundary value problem for the equation of heat conductivity with the Wenzel conjugation condition is studied. It does not fit into the general theory of parabolic initial boundary value problems and belongs to the class of conditionally correct ones. In space of bounded continuous functions by the method of boundary integral equations its classical solvability under some conditions is established. In addition, it is proved that the obtained solution is a Feller semigroup, which represents some homogeneous generalized diffusion process in the area considered here.

Key words and phrases: parabolic potential, boundary integral equation method, Feller semigroup, nonlocal boundary condition.
\end{abstract}

\footnotetext{
${ }^{1}$ Institute of Mathematics, Czestochowa University of Technology, 69 Dabrowskiego str., 42-201, Czestochowa, Poland

2 Ivan Franko National University, 1 Universytetska str., 79000, Lviv, Ukraine

E-mail: bohdan.kopytko@gmail.com (Kopytko B.I.), nandrew183@gmail.com (Novosyadlo A.F.)
}

\section{INTRODUCTION}

The article presents the results of studying one nonclassical initial boundary value problem for the equation of second-order heat conductivity with respect to tangential variables under the Wenzel type conjugation condition $[3,24]$. It is assumed that the source area and its two subdomains, where the conjugation problem is considered, are cylindrical, the basis of which is the strips in the finite-dimensional Euclidean space $\mathbb{R}^{d}$. These results were obtained using the technique developed in $[9,10,17]$ for the case when the bases of the mentioned cylindrical areas coincide with the space $\mathbb{R}^{d}, d \geq 2$, and its half-spaces, respectively.

The following notations are used: $x=\left(x^{\prime}, x_{d}\right)=\left(x_{1}, \ldots, x_{d-1}, x_{d}\right)$ is a point in a finitedimensional Euclidean space $\mathbb{R}^{d}, d \geq 2, x^{\prime}=\left(x_{1}, \ldots, x_{d-1}\right)$ is a point in $\mathbb{R}^{d-1}$ (by $x^{\prime}$ sometimes is denoted the point $\left(x_{1}, \ldots, x_{d-1}, 0\right) ;(x, y)=\sum_{i=1}^{d} x_{i} y_{i}$ for $x, y \in \mathbb{R}^{d} ;\left(x^{\prime}, y^{\prime}\right)=\sum_{i=1}^{d-1} x_{i} y_{i}$ for $x^{\prime}, y^{\prime} \in \mathbb{R}^{d-1} ; \mathcal{D}$ is an area in $\mathbb{R}^{d}$ with boundary $\partial \mathcal{D}=S ;(t, x)=\left(t, x^{\prime}, x_{d}\right)$ is a point in $\mathbb{R}^{d+1}$; $T$ is a fixed positive number; $\mathbb{R}_{\infty}^{d+1}=(0, \infty) \times \mathbb{R}^{d}, \mathbb{R}_{\infty}^{d}=(0, \infty) \times \mathbb{R}^{d-1}, \mathbb{R}_{T}^{d+1}=(0, T) \times \mathbb{R}^{d}$, $\mathbb{R}_{T}^{d}=(0, T) \times \mathbb{R}^{d-1}, \Omega=(0, \infty) \times \mathcal{D}$ and $\Omega_{T}=(0, T) \times \mathcal{D}$ are areas in $\mathbb{R}^{d+1}, \Sigma=(0, \infty) \times S$ and $\Sigma_{T}=(0, T] \times S$ are lateral borders of areas $\Omega$ and $\Omega_{T}$ accordingly; $\bar{Q}$ is the closure of the set $Q ; D_{i}$ the operator of differentiation by variable $x_{i}, i \in\{1, \ldots, d\}$; in particular $\nabla u=$ $\left(D_{1} u, \ldots, D_{d-1} u, D_{d} u\right)=\left(\nabla^{\prime} u, D_{d} u\right)$ is the gradient of function $u ; D_{t}=\frac{\partial}{\partial t}, D_{t}^{r}$, and $D_{x}^{p}$ are symbols respectively of a partial derivative of $t$ of order $r$ and of any partial derivative of $x$ of order $p$, where $r$ and $p$ are integers that are nonnegative; $\delta_{j}^{k}=1$ if $j=k$, and $\delta_{j}^{k}=0$ if $j \neq k ; \triangle_{x}^{\widetilde{x}} f(\cdot, x)=f(\cdot, x)-f(\cdot, \widetilde{x}), \triangle_{t}^{\widetilde{t}} f(t, \cdot)=f(t, \cdot)-f(\widetilde{t}, \cdot) ; C^{l}(\mathcal{D})\left(C^{l}(\overline{\mathcal{D}})\right), l \in\{0,1,2\}$, $\left(C^{0}(\mathcal{D}) \equiv C(\mathcal{D}), C^{0}(\overline{\mathcal{D}}) \equiv C(\overline{\mathcal{D}})\right)$ is a set of continuous functions in $\mathcal{D}$ (in $\overline{\mathcal{D}}$ ), which have continuous derivatives $D_{x}^{p}, p \leq l$, in $\mathcal{D}$ (in $\left.\overline{\mathcal{D}}\right)$, where $\mathcal{D}$ is a subset of $\mathbb{R}^{d} ; C(\Omega)(C(\bar{\Omega}))$ is a

$\mathrm{y} \Delta \mathrm{K} 517.95,519.21$

2010 Mathematics Subject Classification: 60J60, 35K20. 
set of continuous functions in $\Omega$ (in $\bar{\Omega}$ ), where $\Omega$ is a subset of $\mathbb{R}_{\infty}^{d+1} ; C^{1,2}(\Omega)\left(C^{1,2}(\bar{\Omega})\right.$ ) is a set of continuous functions in $\Omega(\bar{\Omega})$, which have continuous derivatives $D_{t}^{r}, D_{x}^{p}, r=1, p \leq 2$, in $\Omega$ (in $\bar{\Omega}$ ); $\mathcal{B}\left(\mathbb{R}^{d}\right)$ is the Banach space of bounded measurable functions $\varphi$ endowed with the norm $\|\varphi\|=\sup _{x \in \mathbb{R}^{d}}|\varphi(x)| ; C_{b}^{l}\left(\mathbb{R}^{d}\right), l \in\{0,1,2\},\left(C_{b}^{0}\left(\mathbb{R}^{d}\right) \equiv C_{b}\left(\mathbb{R}^{d}\right)=C\left(\mathbb{R}^{d}\right) \cap \mathcal{B}\left(\mathbb{R}^{d}\right)\right)$ is the set of all real $l$ times continuously differentiable functions bounded by their derivatives $D_{x}^{p}, p \leq l$; $H^{\lambda}\left(\mathbb{R}^{d-1}\right), \lambda \in(0,1)$, is Hölder space (see [6, Ch. I, §1]).

Various constants, the particular values of which we will mostly not be interested in, are denoted by $C, c$ (with or without indexes). Other designations will be explained where they first appear.

\section{FORMULATION OF WENTZEL INITIAL BOUNDARY VALUE PROBLEM FOR THE HEAT EQUATION AND BASIC ASSUMPTIONS}

Let $\mathcal{D}$ be a strip in the space $\mathbb{R}^{d}, d \geq 2$, which is bounded by hyperplanes

$$
S_{1}=\left\{x \in \mathbb{R}^{d} \mid x_{d}=d_{1}, d_{1}<0\right\}, S_{2}=\left\{x \in \mathbb{R}^{d} \mid x_{d}=d_{2}, d_{2}>0\right\} .
$$

Under such conditions, we assume that $\mathcal{D}$ is separated by a hyperplane

$$
S_{0}=\left\{x \in \mathbb{R}^{d} \mid x_{d}=0\right\}=\mathbb{R}^{d-1}
$$

into two lanes

$$
\mathcal{D}_{1}=\left\{x \in \mathbb{R}^{d} \mid d_{1}<x_{d}<0\right\} \text { and } \mathcal{D}_{2}=\left\{x \in \mathbb{R}^{d} \mid 0<x_{d}<d_{2}\right\},
$$

so $\partial \mathcal{D}=S=S_{1} \cup S_{2}, \partial \mathcal{D}_{1}=S^{(1)}=S_{1} \cup S_{0}, \partial \mathcal{D}_{2}=S^{(2)}=S_{2} \cup S_{0}$. Let $\Omega=(0, \infty) \times \mathcal{D}$, $\Omega_{T}=(0, T) \times \mathcal{D}, \Omega^{(m)}=(0, \infty) \times \mathcal{D}_{m}, \Omega_{T}^{(m)}=(0, T) \times \mathcal{D}_{m}, m \in\{1,2\} ; \Sigma^{(m)}=(0, \infty) \times$ $S_{m}, \Sigma_{T}^{(m)}=(0, T] \times S_{m}, m \in\{0,1,2\}$. Let us denote the normal vector $n(x)=\left(n_{i}(x)\right)_{i=1}^{d}=$ $(0, \ldots, 0,1) \in \mathbb{R}^{d}$ to the $S_{m}$ at the point $x \in S_{m}, m \in\{0,1,2\}$.

Consider Wentzel's initial boundary-value problem: to find a function

$$
u(t, x)= \begin{cases}u_{1}(t, x), & (t, x) \in \bar{\Omega}^{(1)}, \\ u_{2}(t, x), & (t, x) \in \bar{\Omega}^{(2)},\end{cases}
$$

such that in $\Omega^{(m)}$ the function $u_{m}$ is a solution of the equation

$$
D_{t} u_{m}-\frac{1}{2} \Delta u_{m}=0, \quad m \in\{1,2\},
$$

and on the boundary $\Sigma^{(m)}, m \in\{1,2\}$, the function $u_{m}$ satisfies the boundary condition

$$
\left.\left.L_{m} u_{m}\right|_{\Sigma^{(m)}} \equiv \frac{\partial u_{m}(t, x)}{\partial x_{d}}\right|_{x_{d}=d_{m}}=0, \quad m \in\{1,2\},
$$

on the boundary $\Sigma^{(0)}$ the functions $u_{1}$ and $u_{2}$ satisfy the conjugation conditions

$$
\begin{gathered}
\left.\left.L_{01}(u)\right|_{\bar{\Sigma}^{(0)}} \equiv\left(u_{1}(t, x)-u_{2}(t, x)\right)\right|_{x_{d}=0}=0, \\
\left.L_{02}(u)\right|_{\bar{\Sigma}^{(0)}} \equiv\left(\frac{1}{2} \sum_{i, j=1}^{d-1} \beta_{i j}\left(x^{\prime}\right) D_{i} D_{j} u(t, x)\right. \\
+\sum_{i=1}^{d-1} \alpha_{i}\left(x^{\prime}\right) D_{i} u(t, x) \\
\left.-q_{1}\left(x^{\prime}\right) D_{d} u_{1}(t, x)+q_{2}\left(x^{\prime}\right) D_{d} u_{2}(t, x)\right)\left.\right|_{x_{d}=0}=0,
\end{gathered}
$$


and at $t=0$ the following initial conditions are satisfied

$$
\left.u_{m}(t, x)\right|_{t=0}=\varphi(x), \quad x \in \overline{\mathcal{D}}_{m}, m \in\{1,2\} .
$$

Here $\Delta=\sum_{i, j=1}^{d} \frac{\partial^{2}}{\partial x_{i}^{2}}$ is the Laplace operator, $\varphi(x)$ is a given bounded continuous function on $\mathbb{R}^{d}, \beta_{i j}\left(x^{\prime}\right), \alpha_{i}\left(x^{\prime}\right),\{i, j\} \subset\{1, \ldots, d-1\}, q_{m}\left(x^{\prime}\right), m \in\{1,2\}$, are given bounded continuous functions on $\mathbb{R}^{d-1}$ such that

a) $\beta\left(x^{\prime}\right)=\left(\beta_{i j}\left(x^{\prime}\right)\right)_{i, j=1}^{d-1}$ is symmetric and inalienable matrix, in addition there are positive constants $\beta_{1}$ and $\beta_{2}$ such that for all $x^{\prime} \in \mathbb{R}^{d-1}$ and for any real vector $\Theta^{\prime} \in \mathbb{R}^{d-1}$ $\beta_{1}\left|\Theta^{\prime}\right|^{2} \leq\left(\beta\left(x^{\prime}\right) \Theta^{\prime}, \Theta^{\prime}\right) \leq \beta_{2}\left|\Theta^{\prime}\right|^{2}$;

b) $q_{m}\left(x^{\prime}\right) \geq 0, x^{\prime} \in \mathbb{R}^{d-1}, m \in\{1,2\}$, and $\inf _{x^{\prime} \in \mathbb{R}^{d-1}}\left(q_{1}\left(x^{\prime}\right)+q_{2}\left(x^{\prime}\right)\right)>0$;

c) $\beta_{i j}, \alpha_{i}, q_{m} \in H^{\lambda}\left(\mathbb{R}^{d-1}\right), \lambda \in(0,1),\{i, j\} \subset\{1, \ldots, d-1\}, m \in\{1,2\}$.

Problem (2)-(6) with the condition of conjugation consisting the second order derivatives with respect to tangent space variables occurs in the theory of random processes while studying with analytical methods of so-called problem of pasting together of two diffusion processes in an Euclidean space or, equivalently, when building of mathematical models of physical phenomena of diffusion in a medium containing membranes located on fixed surfaces [11,20,21]. In this case the boundary condition (3) and the condition of conjugation (5) are only a few options for the general Wentzel boundary condition for multidimensional diffusion processes $[3,6,23,24]$. We pay attention to the probabilistic meaning of these and other conditions included in the problem (2)-(6). Therefore, if we assume that the obtained solution of the problem (2)-(6) forms a Feller semigroup of operators (we denote it by $T_{t}, t \geq 0$ ), which describes on $\overline{\mathcal{D}}$ some homogeneous Markov process, then performing of the Kolmogorov equation (2) for the function $u(t, x) \equiv T_{t} \varphi(x)$ indicates that this process coincides at the interior points of the domains $\mathcal{D}_{1}$ and $\mathcal{D}_{2}$ with the Wiener process specified there. This process is managed by generating differential operator $L=\frac{1}{2} \Delta$, and the initial condition (6) is consistent with the equality $T_{0}=I$, where $I$ is the identical operator. Condition of conjugation (4) means that the process will be Feller type, and the boundary condition (3) as well as the condition of conjugation (5) responsible for its continuation after entering the diffusing particles on the borders of $\mathcal{D}_{1}$ and $\mathcal{D}_{2}$, where the membranes are located. In particular, the realization for semigroups of boundary conditions (3) means that the process at the points of the surfaces $S_{1}$ and $S_{2}$ behaves like a Brownian motion with reflection. According to (5) process after its release on the $S_{0}$ is carried out in accordance with combinations of effects such as partial reflection, the drift and diffusion along the boundary.

The initial-boundary-value problem (2)-(6) is characterized by the fact that, if the condition of conjugation (5) contains the second order derivatives with respect to tangent space variables, then this problem is not parabolic in the sense that it does not fulfill the so-called complementarity conditions. These conditions in the case of conjugations problems are also called the conditions of compatible covering $[4,7,14,26]$. And this means that this problem does not fit into the class of initial-boundary value problems for parabolic equations and systems, for which the general theory is constructed $[4,5,7,14,22,26]$. Because of this, we call the Wenzel conjugation condition (5) nonclassical one.

The classical solution of the problem (2)-(6) in the space of bounded continuous functions is obtained by using the method of boundary integral equations. We use the potentials constructed by ordinary fundamental solutions of uniformly parabolic operators. It is also proved 
that, using a solution of this problem, one can construct an one-parameter Feller semigroup that corresponds on $\overline{\mathcal{D}}$ to some homogeneous Markov process. Again, we note that the conjugation problem, similar to the problem (2)-(6), has been studied (including in more general formulations) by the potential theory method earlier in $[9,10,17]$ for the case $\mathcal{D}=\mathbb{R}^{d}$ (see also [11,12] for other variants of the general Wenzel boundary condition). In [2], the parabolic variant of Wentzel initial-boundary problem with the boundary condition, containing second order derivatives with respect to tangent space variables, was investigated in the Hölder classes by the local method. We also note the works $[1,6,15,18,23,25]$, which reflect the development of other approaches (both using analytical and direct probabilistic methods) to construct multidimensional diffusion processes under given Wenzel boundary conditions.

\section{THE SOLUTION OF WENTZEL INITIAL BOUNDARY-VALUE PROBLEM}

Let us denote by $g(t, x, y)=q(t, x-y)=q\left(t, x^{\prime}-y^{\prime}, x_{d}-y_{d}\right)\left(t>0,\{x, y\} \subset \mathbb{R}^{d}\right)$ the density of transition probabilities of a $d$-dimensional Wiener process

$$
g(t, x, y)=(2 \pi t)^{-d / 2} \exp \left\{-\frac{|y-x|^{2}}{2 t}\right\}
$$

As we know, this is a fundamental solution for the heat equation

$$
\frac{\partial u(t, x)}{\partial t}-\frac{1}{2} \Delta u(t, x)=0,
$$

that is, a function that has the following properties:

1) it is a continuous function of its arguments;

2) at fixed $y \in \mathbb{R}^{d}$ as a function of $t, x$ in area $(t, x) \in \mathbb{R}_{\infty}^{d+1}$ it satisfies the equation (7);

3) for any function $\varphi \in C_{b}\left(\mathbb{R}^{d}\right)$

$$
\lim _{t \downarrow 0} \int_{\mathbb{R}^{d}} g(t, x, y) \varphi(y) d y=\varphi(x)
$$

for all $t>0, x \in \mathbb{R}^{d}$.

In addition, the function $g(t, x, y)$ is infinitely differentiable for all variables and justifies the inequality

$$
\left|D_{t}^{r} D_{x}^{p} g(t, x, y)\right| \leq C t^{-\frac{d+2 r+p}{2}} \exp \left\{-c \frac{|y-x|^{2}}{t}\right\}
$$

for each domain of the form $t \in(0, T],\{x, y\} \subset \mathbb{R}^{d}$, where $r$ and $p$ are nonnegative integer numbers.

The following relations hold $\left(t \geq 0,\{x, \Theta\} \subset \mathbb{R}^{d}\right)$ :

$$
\begin{aligned}
& \int_{\mathbb{R}^{d}} D_{t}^{r} D_{x}^{p} g(t, x, y) d y= \begin{cases}1, & \text { if } r=0, p=0, t>0, x \in \mathbb{R}^{d}, \\
0, & \text { if } r+p \geq 1, t>0, x \in \mathbb{R}^{d},\end{cases} \\
& \int_{\mathbb{R}^{d}} g(t, x, y)(y-x, \Theta) d y=0, \quad \text { if } t>0,\{x, \Theta\} \subset \mathbb{R}^{d}, \\
& \int_{\mathbb{R}^{d}} g(t, x, y)(y-x, \Theta)^{2} d y=|\Theta|^{2} t, \quad \text { if } t>0,\{x, \Theta\} \subset \mathbb{R}^{d},
\end{aligned}
$$




$$
g(t+s, x, y)=\int_{\mathbb{R}^{d}} g(t, x, z) g(s, z, y) d y, \quad \text { if } t>0, s>0,\{x, y\} \subset \mathbb{R}^{d} .
$$

The correctness of the equalities (10)-(13) can be verified by direct calculations.

The integral on the left side of (8) is the function

$$
u_{0}(t, x)=\int_{\mathbb{R}^{d}} g(t, x, y) \varphi(y) d y .
$$

This function is called the Poisson heat potential with kernel $g$ and density $\varphi$. If $\varphi \in C_{b}\left(\mathbb{R}^{d}\right)$, then potential (14) at $(t, x) \in \mathbb{R}_{\infty}^{d+1}$ is the solution of the equation (7) and satisfies the initial condition

$$
\lim _{t \downarrow 0} u(t, x)=\varphi(x), x \in \mathbb{R}^{d} .
$$
$\mathbb{R}_{T}^{d+1}$ :

In addition, the inequality (9) gives us the following estimate of the function $u_{0}(t, x),(t, x) \in$

$$
\left|D_{t}^{r} D_{x}^{p} u_{0}(t, x)\right| \leq C t^{-\frac{2 r+p}{2}}\|\varphi\| .
$$

In the solution of the problem (2)-(6) also be used the notion of a parabolic potential of a single layer. This concept is associated with some given hypersurface $S$ in $\mathbb{R}^{d}$. We can consider the case when $S$ is a hyperplane in $\mathbb{R}^{d}$ orthogonal to a given orth $n=(0, \ldots, 0,1) \in \mathbb{R}^{d}$. This means that $S=\left\{x \in \mathbb{R}^{d} \mid x_{d}=m\right\}$, where $m$ is a given real constant. The hyperplane $S$ separates $\mathbb{R}^{d}$ into two domains: $\mathcal{D}_{-}=\left\{x \in \mathbb{R}^{d} \mid x_{d}<m\right\}$ and $\mathcal{D}_{+}=\left\{x \in \mathbb{R}^{d} \mid x_{d}>m\right\}$, so $\mathbb{R}^{d}=\mathcal{D}_{-} \cup \mathcal{D}_{+} \cup S$.

So suppose that on the set $(0, \infty) \times S$ there is a continuous function $V(t, x)$ with real values, which in each area of the form $(t, x) \in(0, T] \times S$ allows such an estimate

$$
|V(t, x)| \leq C t^{-\mu}, \quad 0 \leq \mu<1 .
$$

Let for $(t, x) \in \mathbb{R}_{\infty}^{d+1}$

$$
W(t, x)=\int_{0}^{t} d \tau \int_{S} g(t-\tau, x, y) V(\tau, y) d \sigma_{y}
$$

that the inner integral is a surface integral over the variable $y$. Under our assumptions on the hyperplane $S$ the inegral (17) can be written as

$$
W(t, x)=\int_{0}^{t} d \tau \int_{\mathbb{R}^{d-1}} g\left(t-\tau, x^{\prime}-y^{\prime}, x_{d}-m\right) \hat{V}\left(\tau, y^{\prime}\right) d y^{\prime},
$$

where $\hat{V}\left(\tau, y^{\prime}\right)=\left.V(\tau, y)\right|_{y_{d}=m}=V\left(\tau, y^{\prime}, m\right)$. The function $W(t, x)$ is called the parabolic single-layer potential with kernel $g$ and dencity $V$. Note some of its properties (see $[13, \mathrm{Ch}$. IV, $\S 15],[5, \mathrm{Ch} . \mathrm{V}, \S 2],[19, \mathrm{Ch} . \mathrm{XXII}, \S 8])$. If $V(t, x)$ satisfies the inequality (16), then the integral (18) exists and it is a continuous function on the set of variables $t>0, x \in \mathbb{R}^{d}$. In addition, the function $W(t, x)$ in each area $(t, x) \in(0, \infty) \times \mathcal{D}_{-}$and $(t, x) \in(0, \infty) \times \mathcal{D}_{+}$satisfies the equation (7) and the initial condition

$$
\lim _{t \downarrow 0} W(t, x)=0, \quad x \in \mathbb{R}^{d} \backslash S,
$$

and provided that in (16) the power $\mu \in[0,1 / 2)$ zero initial condition (19) is satisfied for $W(t, x)$ for all $x \in \mathbb{R}^{d}$. The case of $\mu=1 / 2$ in each situation needs to be investigated separately.

Note that with some additional assumptions on the smoothness of the density $V$, not only the function $W$, but also its partial derivatives by tangent variables of the first and second 
orders will change continuously when passing through the surface $S$, that is, $D_{i} W$ and $D_{i} D_{j} W$, $\{i, j\} \subset\{1, \ldots, d-1\}$.

As for the first-order derivative of the function $W$ in the normal direction to $S$, its behavior at $x \in S$, is determined by the statement, which is known in the literature as the theorem for the jump of a conormal derivative of the single-layer potential. In the case under consideration, this theorem states (see, for example, [19, Ch.XXII, §8, Theorem 2], [21, Ch.3, §3, Formula (3.45)]): if $S=\left\{x \in \mathbb{R}^{d} \mid x_{d}=m\right\}$, and the function $V(t, x)$ in the formula (17) is continuous in the domain $(t, x) \in(0, \infty) \times S$ and satisfy of the estimate (16), then

$$
\lim _{x_{d} \rightarrow m \pm} \frac{\partial W(t, x)}{\partial x_{d}}=\mp \hat{V}\left(t, x^{\prime}\right), \quad\left(t, x^{\prime}\right) \in \mathbb{R}_{\infty}^{d} .
$$

In addition, the minus sign on the right-hand side of (20) should be taken when $x=\left(x^{\prime}, x_{d}\right) \in$ $\mathbb{R}^{d}$ approaches to $\left(x^{\prime}, m\right) \in S$ from the side of the area $\mathcal{D}_{+}$, and the plus sign should be taken when $x=\left(x^{\prime}, x_{d}\right) \in \mathbb{R}^{d}$ approaches to $\left(x^{\prime}, m\right) \in S$ from the side of the area $\mathcal{D}_{-}$.

Theorem 1 (On the existence and uniqueness of Wenzel's initial boundary-value problem). Suppose that conditions (a)-(c) hold. Then, if $\varphi \in C_{b}\left(\mathbb{R}^{d}\right)$, the boundary-value problem (2)(6) has a unique classical solution continuous in the closed domain $\bar{\Omega}$.

Proof. To solve the initial boundary value problem (2)-(6), we apply the method of heat and parabolic potentials, which are constructed using ordinary fundamental solutions of uniform parabolic operators. Based on the properties of the fundamental solution of the heat equation $g$ and the resulting heat and parabolic potentials, we will search for each of the functions $u_{1}$ and $u_{2}$ from (1) as the sum of the Poisson potential (14) and the single-layer potentials associated with the hyperplanes $S_{k}, k \in\{0,1,2\}$ :

$$
u_{m}(t, x)=u_{0}(t, x)+u_{m 1}(t, x)+u_{m 2}(t, x),(t, x) \in \bar{\Omega}^{(m)}, m \in\{1,2\},
$$

where

$$
\begin{gathered}
u_{0}(t, x)=\int_{\mathbb{R}^{d}} g(t, x, y) \varphi(y) d y, \\
u_{m 1}(t, x)=\int_{0}^{t} d \tau \int_{S_{0}} g(t-\tau, x, y) V_{m}(\tau, y) d \sigma_{y}=\int_{0}^{t} d \tau \int_{\mathbb{R}^{d-1}} g\left(t-\tau, x^{\prime}-y^{\prime}, x_{d}\right) \hat{V}_{m}\left(\tau, y^{\prime}\right) d y^{\prime}, \\
u_{m 2}(t, x)=\int_{0}^{t} d \tau \int_{S_{m}} g(t-\tau, x, y) V_{m+2}(\tau, y) d \sigma_{y} \\
=\int_{0}^{t} d \tau \int_{\mathbb{R}^{d-1}} g\left(t-\tau, x^{\prime}-y^{\prime}, x_{d}-d_{m}\right) \hat{V}_{m+2}\left(\tau, y^{\prime}\right) d y^{\prime}, \quad m \in\{1,2\},
\end{gathered}
$$

and $V_{m}(\tau, y)=V_{m}\left(\tau, y^{\prime}, 0\right)=\hat{V}_{m}\left(\tau, y^{\prime}\right), V_{m+2}(\tau, y)=V_{m+2}\left(\tau, y^{\prime}, d_{m}\right)=\hat{V}_{m+2}\left(\tau, y^{\prime}\right), m \in$ $\{1,2\}$, are unknown functions which have to be determined.

The unknown functions $V_{m}$ and $V_{m+2}, m \in\{1,2\}$, are found from the boundary conditions (3) and the conjugation conditions (4), (5). In particular, using the boundary conditions (3), using the jump formula (20) applied only to the potentials of the single layer $u_{m 2}, m \in\{1,2\}$, we obtain

$$
\hat{V}_{m+2}\left(t, x^{\prime}\right)=\hat{\psi}_{m}\left(t, x^{\prime}\right)+\int_{0}^{t} d \tau \int_{\mathbb{R}^{d-1}} \hat{K}_{m}\left(t-\tau, x^{\prime}, y^{\prime}\right) \hat{V}_{m}\left(\tau, y^{\prime}\right) d y^{\prime}, \quad m \in\{1,2\},
$$

where

$$
\hat{\psi}_{m}\left(t, x^{\prime}\right)=\left.(-1)^{m-1} \frac{\partial u_{0}(t, x)}{\partial x_{d}}\right|_{x_{d}=d_{m}}, \quad m \in\{1,2\}
$$




$$
\hat{K}_{m}\left(t-\tau, x^{\prime}, y^{\prime}\right)=\left.\frac{\partial g\left(t-\tau, x^{\prime}-y^{\prime}, x_{d}\right)}{\partial x_{d}}\right|_{x_{d}=d_{m}}, \quad m \in\{1,2\} .
$$

For functions $\hat{\psi}_{m}\left(t, x^{\prime}\right)\left(\left(t, x^{\prime}\right) \in \mathbb{R}_{T}^{d}\right)$ and $\hat{K}_{m}\left(t-\tau, x^{\prime}, y^{\prime}\right)\left(0 \leq \tau<t \leq T,\left\{x^{\prime}, y^{\prime}\right\} \subset \mathbb{R}^{d-1}\right)$ the following estimates come true:

$$
\begin{gathered}
\left|\hat{\psi}_{m}\left(t, x^{\prime}\right)\right| \leq C\|\varphi\| t^{-1 / 2}, \quad m \in\{1,2\}, \\
\left|\hat{K}_{m}\left(t-\tau, x^{\prime}, y^{\prime}\right)\right| \leq C \exp \left\{-c \frac{\left|x^{\prime}-y^{\prime}\right|^{2}}{t-\tau}\right\}, \quad m \in\{1,2\} .
\end{gathered}
$$

Indeed, the inequality (23) follows directly from (15) at $r=0, p=1$, and the estimate (24) is a simple consequence of the relation

$$
\left.\frac{\partial g\left(t-\tau, x^{\prime}-y^{\prime}, x_{d}\right)}{\partial x_{d}}\right|_{x_{d}=d_{m}}=-\frac{d_{m}}{(2 \pi)^{d / 2}(t-\tau)^{\frac{2+d}{2}}} \exp \left\{-\frac{d_{m}^{2}}{2(t-\tau)}\right\} \exp \left\{-\frac{\left|x^{\prime}-y^{\prime}\right|^{2}}{2(t-\tau)}\right\},
$$

for $\left|d_{m}\right|>0, m \in\{1,2\}$, and inequality

$$
\sigma^{v} e^{-c \sigma} \leq \text { const } \quad(0 \leq \sigma<\infty, 0 \leq v<\infty) .
$$

The equations (22) are integral Volterra equations of the second kind, solved with respect to the functions $\hat{V}_{3}$ and $\hat{V}_{4}$.

Let us consider the conjugation condition (4). Substituting the expressions for $u_{1}\left(t, x^{\prime}, 0\right)$ and $u_{2}\left(t, x^{\prime}, 0\right)$ from $(21)$, we find

$$
\begin{aligned}
\int_{0}^{t} d \tau & \int_{\mathbb{R}^{d-1}} g\left(t-\tau, x^{\prime}-y^{\prime}, 0\right)\left[\hat{V}_{1}\left(\tau, y^{\prime}\right)-\hat{V}_{2}\left(\tau, y^{\prime}\right)\right] d y^{\prime} \\
\quad & =\sum_{j=1}^{2}(-1)^{j} \int_{0}^{t} d \tau \int_{\mathbb{R}^{d-1}} g\left(t-\tau, x^{\prime}-y^{\prime},-d_{m}\right) \hat{V}_{j+2}\left(\tau, y^{\prime}\right) d y^{\prime}, \quad\left(t, x^{\prime}\right) \in \mathbb{R}_{\infty}^{d} .
\end{aligned}
$$

The equation (25) is integral Volterra equation of the first kind. In order to regularize this equation, we apply the integral-differential operator $\mathcal{E}_{1}$ to both parts of this equation, which operates by the rule

$$
\mathcal{E}_{1} f\left(t, x^{\prime}\right)=\left.\sqrt{2 / \pi}\left\{\frac{\partial}{\partial t} \int_{0}^{t}(t-\tau)^{-1 / 2} d \tau \int_{\mathbb{R}^{d-1}} h\left(\hat{t}-\tau, x^{\prime}-y^{\prime}\right) f\left(\tau, y^{\prime}\right) d y^{\prime}\right\}\right|_{\hat{t}=t},
$$

where $h$ denotes the fundamental solution of $D_{t}-\frac{1}{2} \Delta^{\prime}, \Delta^{\prime}=\sum_{i=1}^{d-1} \frac{\partial^{2}}{\partial x_{i}^{2}}$ :

$$
h\left(t, x^{\prime}, y^{\prime}\right)=(2 \pi t)^{-\frac{d-1}{2}} \exp \left\{-\frac{\left|x^{\prime}-y^{\prime}\right|^{2}}{2 t}\right\}, \quad t>0,\left\{x^{\prime}, y^{\prime}\right\} \subset \mathbb{R}^{d-1}
$$

As a result, we obtain an equivalent Volterra integral equation of the second kind, solved with respect to $\hat{V}_{1}\left(t, x^{\prime}\right)-\hat{V}_{2}\left(t, x^{\prime}\right)$ :

$$
\hat{V}_{1}\left(t, x^{\prime}\right)-\hat{V}_{2}\left(t, x^{\prime}\right)=\left.\sum_{j=1}^{2} \int_{0}^{t} d \tau \int_{\mathbb{R}^{d-1}} \frac{\partial g\left(t-\tau, x^{\prime}-y^{\prime}, x_{d}-d_{m}\right)}{\partial x_{d}}\right|_{x_{d}=0} \hat{V}_{j+2}\left(\tau, y^{\prime}\right) d y^{\prime} .
$$

In the equation (26), as in (22), the kernels of the single-layer potentials allow the estimate (24). 
Note that the equivalence of the equations (26) and (25) in this case is sufficiently easy to justify by applying to both parts of these equations the Fourier and Laplace transforms for the variables $x^{\prime}$ and $t$, respectively.

So we have to use the conjugation condition (5). For this at first, we use the jump formula (20) and the notations

$$
\begin{aligned}
\beta_{i j}^{(0)}\left(x^{\prime}\right)= & \frac{\beta_{i j}\left(x^{\prime}\right)}{q_{1}\left(x^{\prime}\right)+q_{2}\left(x^{\prime}\right)}, \quad \alpha_{i}^{(0)}\left(x^{\prime}\right)=\frac{\alpha_{i}\left(x^{\prime}\right)}{q_{1}\left(x^{\prime}\right)+q_{2}\left(x^{\prime}\right)}, \quad\{i, j\} \subset\{1, \ldots, d-1\}, \\
q\left(x^{\prime}\right)= & \frac{q_{2}\left(x^{\prime}\right)-q_{1}\left(x^{\prime}\right)}{q_{1}\left(x^{\prime}\right)+q_{2}\left(x^{\prime}\right)}, \quad\left|q\left(x^{\prime}\right)\right| \leq 1, \\
\Theta\left(t, x^{\prime}\right)= & \sum_{j=1}^{2} \frac{1}{2}\left[1+(-1)^{j} q\left(x^{\prime}\right)\right] \hat{V}_{j}\left(t, x^{\prime}\right) \\
& \quad-\left.\sum_{j=1}^{2} \int_{0}^{t} d \tau \int_{\mathbb{R}^{d-1}} \frac{1}{2}\left[q\left(x^{\prime}\right)+(-1)^{j}\right] \frac{\partial g\left(t-\tau, x^{\prime}-y^{\prime}, x_{d}-d_{j}\right)}{\partial x_{d}}\right|_{x_{d}=0} \hat{V}_{j+2}\left(\tau, y^{\prime}\right) d y^{\prime} \\
& \quad-\left.q\left(x^{\prime}\right) D_{d} u_{0}(t, x)\right|_{x_{d}=0}-u\left(t, x^{\prime}, 0\right) .
\end{aligned}
$$

For $m \in\{1,2\}$ we find the following image

$$
\left.L_{0}^{\prime} u\right|_{\Sigma^{(0)}} \equiv \frac{1}{2} \sum_{i, j=1}^{d-1} \beta_{i j}^{(0)}\left(x^{\prime}\right) D_{i} D_{j} u\left(t, x^{\prime}, 0\right)+\sum_{i=1}^{d-1} \alpha_{i}^{(0)}\left(x^{\prime}\right) D_{i} u\left(t, x^{\prime}, 0\right)-u_{m}\left(t, x^{\prime}, 0\right)=\Theta\left(t, x^{\prime}\right) .
$$

The coefficients $\beta_{i j}^{(0)}\left(x^{\prime}\right)$ and $\alpha_{i}^{(0)}\left(x^{\prime}\right)$ are elements of the matrix $\beta_{0}\left(x^{\prime}\right)=\left(\beta_{i j}^{(0)}\left(x^{\prime}\right)\right)_{i, j=1}^{d-1}$ and the coordinates of the vector $\alpha_{0}\left(x^{\prime}\right)=\left(\alpha_{i}^{(0)}\left(x^{\prime}\right)\right)_{i=1}^{d-1}$ respectively. By $\beta_{0}^{-1}\left(x^{\prime}\right)$ we denote the matrix inversed to the matrix $\beta_{0}\left(x^{\prime}\right)$.

We can consider (27) as a stand-alone elliptic equation for $S_{0}$, in which we interpret the variable $t$ as a parameter. From the conditions (a)-(c) it follows that the operator $L_{0}^{\prime}$ is uniformly elliptic and there exists its fundamental solution (see [16, Ch.III, §20], [8]), which we denote by $\Gamma\left(x^{\prime}, z^{\prime}\right),\left\{x^{\prime}, z^{\prime}\right\} \subset \mathbb{R}^{d-1}$. In this case, the function $\Gamma\left(x^{\prime}, z^{\prime}\right)$ can be expressed by the formula

$$
\Gamma\left(x^{\prime}, z^{\prime}\right)=\int_{0}^{\infty} e^{-s} G\left(s, x^{\prime}, z^{\prime}\right) d s,
$$

where $G\left(s, x^{\prime}, z^{\prime}\right)\left(s>0,\left\{x^{\prime}, z^{\prime}\right\} \subset \mathbb{R}^{d-1}\right)$ is fundamental solution of uniform parabolic operator

$$
L^{\prime}=\frac{1}{2} \sum_{i, j=1}^{d-1} \beta_{i j}^{(0)}\left(x^{\prime}\right) D_{i} D_{j}+\sum_{i=1}^{d-1} \alpha_{i}^{(0)}\left(x^{\prime}\right) D_{i}-D_{s} .
$$

We use some properties of the function $G\left(s, x^{\prime}, z^{\prime}\right)$ (see [13, Ch.IV, §11-12], [20, Ch.2, §5]). In particular, we will need the following representation of the fundamental solution $G\left(s, x^{\prime}, z^{\prime}\right)$

$$
G\left(s, x^{\prime}, z^{\prime}\right)=G_{0}\left(s, x^{\prime}, z^{\prime}\right)+G_{1}\left(s, x^{\prime}, z^{\prime}\right),
$$

where

$$
\begin{gathered}
G_{0}\left(s, x^{\prime}, z^{\prime}\right)=G_{0}^{\left(z^{\prime}\right)}\left(s, x^{\prime}-z^{\prime}\right)=(2 \pi s)^{-\frac{d-1}{2}}\left(\operatorname{det}\left(\beta_{0}\left(z^{\prime}\right)\right)\right)^{-1 / 2} \exp \left\{-\frac{1}{2 s}\left(\beta_{0}^{-1}\left(z^{\prime}\right)\left(z^{\prime}-x^{\prime}\right), z^{\prime}-x^{\prime}\right)\right\}, \\
G_{1}\left(s, x^{\prime}, z^{\prime}\right)=\int_{0}^{s} d \tau \int_{\mathbb{R}^{d-1}} G_{0}\left(s-\tau, x^{\prime}, y^{\prime}\right) Q_{0}\left(\tau, y^{\prime}, z^{\prime}\right) d y^{\prime},
\end{gathered}
$$


$Q_{0}$ is a solution of some integral Volterra equation of the second kind. In addition, the functions $G$ and $G_{1}$ satisfy such inequalities

$$
\begin{gathered}
\left|D_{s}^{r} D_{x^{\prime}}^{p} G\left(s, x^{\prime}, z^{\prime}\right)\right| \leq C s^{-\frac{(d-1)+2 r+p}{2}} \exp \left\{-c \frac{\left|x^{\prime}-z^{\prime}\right|^{2}}{s}\right\}, \\
\left|D_{s}^{r} D_{x^{\prime}}^{p} G_{1}\left(s, x^{\prime}, z^{\prime}\right)\right| \leq C s^{-\frac{(d-1)+2 r+p-\lambda}{2}} \exp \left\{-c \frac{\left|x^{\prime}-z^{\prime}\right|^{2}}{s}\right\},
\end{gathered}
$$

where $2 r+p \leq 2, s \in(0, T],\left\{x^{\prime}, y^{\prime}\right\} \subset \mathbb{R}^{d-1}, C=C_{T}<\infty$, if $T<\infty$.

The inequality (28) holds for $G_{0}$ if $r$ and $p$ are any nonnegative integers.

If the expressions $D_{s}^{r} D_{x^{\prime}}^{p} G\left(s, x^{\prime}, z^{\prime}\right)$ and $D_{s}^{r} D_{x^{\prime}}^{p} G_{1}\left(s, x^{\prime}, z^{\prime}\right)$ be evaluated by the factor $e^{-\mu s}$, $\mu>0$, we can show that the inequalities (28) and (29) will be correct for $s \in(0, \infty),\left\{x^{\prime}, z^{\prime}\right\} \subset$ $\mathbb{R}^{d-1}$ (that is, in this case $C$ becomes independent of $T$ ).

We will also use estimates of the form (13.2) and (13.3) from [13, Ch.IV, §13] (applied to differences $\Delta_{x^{\prime}}^{\tilde{x}^{\prime}}\left(D_{s}^{r} D_{x^{\prime}}^{p} G\left(s, x^{\prime}, z^{\prime}\right)\right)$ and $\Delta_{t}^{\tilde{t}}\left(D_{s}^{r} D_{x^{\prime}}^{p} G\left(s, x^{\prime}, z^{\prime}\right)\right)$ respectively), as well as the relations (2.38), (2.39) from [20, Ch.2, §2] (they, like to the equations (10) and (13) with obvious changes, will be applied to the potentials generated by the fundamental solution $G$ ).

Next, assuming that $\Theta\left(t, x^{\prime}\right)$ with (27) as a function of the variable $x^{\prime}$ is bounded and Hölder continuous, we have that a unique solution of the equation (27) for $\left(t, x^{\prime}\right) \in \mathbb{R}_{\infty}^{d}$ can be presented in the form

$$
u\left(t, x^{\prime}, 0\right)=-\int_{\mathbb{R}^{d-1}} \Gamma\left(x^{\prime}, z^{\prime}\right) \Theta\left(t, z^{\prime}\right) d z^{\prime}=-\int_{0}^{\infty} e^{-s} d s \int_{\mathbb{R}^{d-1}} G\left(s, x^{\prime}, z^{\prime}\right) \Theta\left(t, z^{\prime}\right) d z^{\prime} .
$$

So, in addition to the formula (21), where we need to take $(t, x)=\left(t, x^{\prime}, 0\right)$, we found for the function $u\left(t, x^{\prime}, 0\right)$ the relation (30). If we equate their right parts, given (26), we obtain the following two integral equations that can be solved with respect to $\hat{V}_{m}, m \in\{1,2\}$ :

$$
\begin{aligned}
& \int_{0}^{t} d \tau \int_{\mathbb{R}^{d-1}}\left[g\left(t-\tau, x^{\prime}-y^{\prime}, 0\right)\right.\left.-\int_{0}^{\infty} e^{-s} d s \int_{\mathbb{R}^{d-1}} G\left(s, x^{\prime}, z^{\prime}\right) g\left(t-\tau, z^{\prime}-y^{\prime}, 0\right) d z^{\prime}\right] \hat{V}_{m}\left(\tau, y^{\prime}\right) d y^{\prime} \\
&+\int_{0}^{\infty} e^{-s} d s \int_{\mathbb{R}^{d-1}} G\left(s, x^{\prime}, z^{\prime}\right) \hat{V}_{m}\left(t, z^{\prime}\right) d z^{\prime} \\
&=\sum_{j=1}^{2} \int_{0}^{t} d \tau \int_{\mathbb{R}^{d-1}} K_{j}^{(m)}\left(t-\tau, x^{\prime}, y^{\prime}\right) \hat{V}_{j+2}\left(\tau, y^{\prime}\right) d y^{\prime}+\psi\left(t, x^{\prime}\right), \quad\left(t, x^{\prime}\right) \in \mathbb{R}_{\infty}^{d}
\end{aligned}
$$

where

$$
\begin{aligned}
& K_{j}^{(m)}\left(t-\tau, x^{\prime}, y^{\prime}\right)=\int_{0}^{\infty} e^{-s} d s \int_{\mathbb{R}^{d-1}} G\left(s, x^{\prime}, z^{\prime}\right)\left[g\left(t-\tau, z^{\prime}-y^{\prime},-d_{j}\right)+q\left(z^{\prime}\right)\right. \\
& \left.\quad \times\left.\frac{\partial g\left(t-\tau, z^{\prime}-y^{\prime}, z_{d}-d_{j}\right)}{\partial z_{d}}\right|_{z_{d}=0}\right] d z^{\prime}-g\left(t-\tau, x^{\prime}-y^{\prime},-d_{m}\right), \quad \text { if } j=m,\{j, m\} \subset\{1,2\}, \\
& K_{j}^{(m)}\left(t-\tau, x^{\prime}, y^{\prime}\right)=\int_{0}^{\infty} e^{-s} d s \int_{\mathbb{R}^{d-1}} G\left(s, x^{\prime}, z^{\prime}\right)\left[q\left(z^{\prime}\right)+(-1)^{m-1}\right] \\
& \times\left.\frac{\partial g\left(t-\tau, z^{\prime}-y^{\prime}, z_{d}-d_{j}\right)}{\partial z_{d}}\right|_{z_{d}=0} d z^{\prime}, \quad \text { if } j \neq m,\{j, m\} \subset\{1,2\}, \\
& \psi\left(t, x^{\prime}\right)=\int_{0}^{\infty} e^{-s} d s \int_{\mathbb{R}^{d-1}} G\left(s, x^{\prime}, z^{\prime}\right)\left[\left.q\left(z^{\prime}\right) D_{d} u_{0}(t, z)\right|_{z_{d}=0}+\left(u_{0}\left(t, z^{\prime}, 0\right)-u_{0}\left(t, x^{\prime}, 0\right)\right)\right] d z^{\prime} .
\end{aligned}
$$


The equation (31) is an integral equation of the first kind of Volterra-Fredholm type. In order to regularize it, we introduce the integro-differential operator $\mathcal{E}_{2}$, which operates by the rule

$$
\begin{aligned}
\mathcal{E}_{2} f\left(t, x^{\prime}\right) & =\sqrt{2 / \pi}\left\{\frac { \partial } { \partial t } \int _ { 0 } ^ { t } ( t - \tau ) ^ { - 1 / 2 } d \tau \int _ { \mathbb { R } ^ { d - 1 } } f ( \tau , y ^ { \prime } ) d y ^ { \prime } \left[h\left(\hat{t}-\tau, x^{\prime}-y^{\prime}\right)\right.\right. \\
& \left.\left.+\int_{0}^{\infty}\left(1-\frac{u}{t-\tau}\right) \exp \left\{-\frac{u^{2}}{2(t-\tau)}\right\} d u \int_{\mathbb{R}^{d-1}} h\left(\hat{t}-\tau, x^{\prime}-v^{\prime}\right) G\left(u, v^{\prime}, y^{\prime}\right) d v^{\prime}\right]\right\}\left.\right|_{\hat{t}=t} .
\end{aligned}
$$

The application of the operator $\mathcal{E}_{2}$ to both parts of each equations (31) leads us to a system of Volterra type II equations solved with respect to $\hat{V}_{m}, m \in\{1,2\}$ :

$$
\begin{aligned}
& \hat{V}_{m}\left(t, x^{\prime}\right)=\int_{0}^{t} d \tau \int_{\mathbb{R}^{d-1}} \hat{K}\left(t-\tau, x^{\prime}, y^{\prime}\right) \hat{V}_{m}\left(\tau, y^{\prime}\right) d y^{\prime} \\
& \quad+\sum_{j=1}^{2} \int_{0}^{t} d \tau \int_{\mathbb{R}^{d-1}} \hat{K}_{j}^{(m)}\left(t-\tau, x^{\prime}, y^{\prime}\right) \hat{V}_{j+2}\left(\tau, y^{\prime}\right) d y^{\prime}+\hat{\psi}\left(t, x^{\prime}\right),\left(t, x^{\prime}\right) \in \mathbb{R}_{\infty}^{d}, m \in\{1,2\} .
\end{aligned}
$$

Here $\hat{\psi}\left(t, x^{\prime}\right)=\mathcal{E}_{2} \psi\left(t, x^{\prime}\right)$, and kernels $\hat{K}\left(t-\tau, x^{\prime}, y^{\prime}\right)$ and $\hat{K}_{j}^{(m)}\left(t-\tau, x^{\prime}, y^{\prime}\right),\{j, m\} \subset\{1,2\}$, are denoted by formulas:

$$
\begin{aligned}
& \hat{K}\left(t-\tau, x^{\prime}, y^{\prime}\right)= \sqrt{\frac{2}{\pi}}\left\{\frac{\partial}{\partial t} \int_{\tau}^{t} d \theta \int_{\mathbb{R}^{d-1}} g\left(\theta-\tau, z^{\prime}-y^{\prime}, 0\right) d z^{\prime} \int_{0}^{\infty} u \cdot(t-\theta)^{-3 / 2} \exp \left\{-\frac{u^{2}}{2(t-\theta)}\right\} d u\right. \\
&\left.\times \int_{\mathbb{R}^{d-1}} h\left(\hat{t}-\theta, x^{\prime}-v^{\prime}\right)\left[G\left(u, v^{\prime}, z^{\prime}\right)-G_{0}^{\left(x^{\prime}\right)}\left(u, v^{\prime}-z^{\prime}\right)\right] d v^{\prime}\right\}\left.\right|_{\hat{t}=t} \\
&- \sqrt{\frac{2}{\pi}}\left\{\frac{\partial}{\partial t} \int_{0}^{\infty}(t-\tau)^{-1 / 2} \exp \left\{-\frac{u^{2}}{2(t-\tau)}\right\} d u\right. \\
&\left.\times \int_{\mathbb{R}^{d-1}} h\left(\hat{t}-\tau, x^{\prime}-v^{\prime}\right)\left[G\left(u, v^{\prime}, y^{\prime}\right)-G_{0}^{\left(x^{\prime}\right)}\left(u, v^{\prime}-y^{\prime}\right)\right] d v^{\prime}\right\}\left.\right|_{\hat{t}=t}, \\
& \hat{K}_{j}^{(m)}\left(t-\tau, x^{\prime}, y^{\prime}\right)=\hat{K}_{j 1}^{(m)}\left(t-\tau, x^{\prime}, y^{\prime}\right)+\hat{K}_{j 2}^{(m)}\left(t-\tau, x^{\prime}, y^{\prime}\right),
\end{aligned}
$$

where

$$
\begin{aligned}
\hat{K}_{j 1}^{(m)}\left(t-\tau, x^{\prime}, y^{\prime}\right) & =\sqrt{\frac{2}{\pi}}\left\{\frac{\partial}{\partial t} \int_{\tau}^{t} d \theta \int_{\mathbb{R}^{d-1}}\left[q\left(z^{\prime}\right)+\frac{1}{2}\left((-1)^{m-1}+(-1)^{j}\right)\right]\right. \\
& \times\left.\frac{\partial g\left(\theta-\tau, z^{\prime}-y^{\prime}, z_{d}-d_{j}\right)}{\partial z_{d}}\right|_{z_{d}=0} d z^{\prime} \int_{0}^{\infty}(t-\theta)^{-1 / 2} \exp \left\{-\frac{u^{2}}{2(t-\theta)}\right\} d u \\
& \left.\times \int_{\mathbb{R}^{d-1}} h\left(\hat{t}-\theta, x^{\prime}-v^{\prime}\right) G\left(u, v^{\prime}, z^{\prime}\right) d v^{\prime}\right\}\left.\right|_{\hat{t}=t} ^{\prime},\{m, j\} \subset\{1,2\}, \\
\hat{K}_{j 2}^{(m)}\left(t-\tau, x^{\prime}, y^{\prime}\right) & =0, \quad \text { if }\{m, j\} \subset\{1,2\} \text { and } m \neq j,
\end{aligned}
$$

and

$$
\begin{aligned}
\hat{K}_{j 2}^{(m)}\left(t-\tau, x^{\prime}, y^{\prime}\right)=-\left.\frac{\partial g\left(t-\tau, x^{\prime}-y^{\prime}, x_{d}-d_{m}\right)}{\partial x_{d}}\right|_{x_{d}=0} \\
+\sqrt{\frac{2}{\pi}}\left\{\frac{\partial}{\partial t} \int_{\tau}^{t} d \theta \int_{\mathbb{R}^{d-1}} g\left(\theta-\tau, z^{\prime}-y^{\prime},-d_{j}\right) d z^{\prime} \int_{0}^{\infty} u \cdot(t-\theta)^{-3 / 2} \exp \left\{-\frac{u^{2}}{2(t-\theta)}\right\} d u\right. \\
\left.\quad \times \int_{\mathbb{R}^{d-1}} h\left(\hat{t}-\theta, x^{\prime}-v^{\prime}\right) G\left(u, v^{\prime}, z^{\prime}\right) d v^{\prime}\right\}\left.\right|_{\hat{t}=t}, \quad \text { if }\{m, j\} \subset\{1,2\} \text { and } m=j .
\end{aligned}
$$


Using the properties of the fundamental solutions $g, h$ and $G$ we prove that for kernels $\hat{K}\left(t-\tau, x^{\prime}, y^{\prime}\right)$ and $\hat{K}_{j}^{(m)}\left(t-\tau, x^{\prime}, y^{\prime}\right)$ (where $\{j, m\} \subset\{1,2\}, 0 \leq \tau<t \leq T, \quad\left(x^{\prime}, y^{\prime}\right) \subset \mathbb{R}^{d-1}$, $0<\gamma<\lambda)$ next estimates hold:

$$
\begin{gathered}
\left|\hat{K}\left(t-\tau, x^{\prime}, y^{\prime}\right)\right| \leq C(t-\tau)^{-1+\frac{\lambda-\gamma}{4} \Phi_{c, \gamma}\left(t-\tau, x^{\prime}, y^{\prime}\right),} \\
\left|\hat{K}_{j}^{(m)}\left(t-\tau, x^{\prime}, y^{\prime}\right)\right| \leq C \cdot \Phi_{c, \gamma}\left(t-\tau, x^{\prime}, y^{\prime}\right)+ \begin{cases}C \cdot \exp \left\{-c \frac{\left|x^{\prime}-y^{\prime}\right|^{2}}{t-\tau}\right\}, & \text { if } m=j, \\
0, & \text { if } m \neq j,\end{cases}
\end{gathered}
$$

where by $\Phi_{c, \gamma}\left(t-\tau, x^{\prime}, y^{\prime}\right)$ we denote the integral

$$
\Phi_{c, \gamma}\left(t-\tau, x^{\prime}, y^{\prime}\right)=\int_{0}^{\infty} u^{-1+\gamma / 2} \exp \left\{-c \frac{u^{2}}{t-\tau}\right\}(t-\tau+u)^{-\frac{d-1}{2}} \exp \left\{-c \frac{\left|x^{\prime}-y^{\prime}\right|^{2}}{t-\tau+u}\right\} d u
$$

Let us analyze in more details the function $\hat{\psi}\left(t, x^{\prime}\right)$ in (32). For it, using the properties of the fundamental solutions $g$ and $G$, we find the following representation:

$$
\begin{aligned}
& \sqrt{\frac{\pi}{2}} \hat{\psi}\left(t, x^{\prime}\right)=\frac{1}{2} \int_{0}^{t}(t-\tau)^{-3 / 2} d \tau \int_{\mathbb{R}^{d-1}} h\left(t-\tau, x^{\prime}-y^{\prime}\right) \\
& \times\left[\Delta_{y^{\prime}}^{x^{\prime}} u_{0}\left(t, y^{\prime}, 0\right)-\left(y^{\prime}-x^{\prime}, \nabla^{\prime} u_{0}\left(\tau, x^{\prime}, 0\right)\right)\right] d y^{\prime} \\
& -\int_{0}^{t} d \tau \int_{\mathbb{R}^{d-1}} \Delta_{y^{\prime}}^{x^{\prime}} u_{0}\left(\tau, y^{\prime}, 0\right) d y^{\prime} \int_{0}^{\infty} D_{u} D_{t}\left((t-\tau)^{-1 / 2}\right. \\
& \left.\times \exp \left\{-\frac{u^{2}}{2(t-\tau)}\right\}\right) d u \int_{\mathbb{R}^{d-1}} h\left(t-\tau, x^{\prime}-v^{\prime}\right)\left[\Delta_{v^{\prime}}^{x^{\prime}} G\left(u, v^{\prime}, y^{\prime}\right)\right] d v^{\prime} \\
& -\int_{0}^{t} d \tau \int_{\mathbb{R}^{d-1}} \Delta_{y^{\prime}}^{x^{\prime}} D_{t} u_{0}\left(t-\tau, y^{\prime}, 0\right) d y^{\prime} \int_{0}^{\infty} D_{u}\left(\tau^{-1 / 2} \exp \left\{-\frac{u^{2}}{2 \tau}\right\}\right) G\left(u, x^{\prime}, y^{\prime}\right) d u \\
& +\left.\int_{0}^{t} d \tau \int_{\mathbb{R}^{d-1}} q\left(y^{\prime}\right) \frac{\partial u_{0}(\tau, y)}{\partial y_{d}}\right|_{y_{d}=0} d y^{\prime} \int_{0}^{\infty} D_{t}\left((t-\tau)^{-1 / 2} \exp \left\{-\frac{u^{2}}{2(t-\tau)}\right\}\right) d u \\
& \times \int_{\mathbb{R}^{d-1}} h\left(t-\tau, x^{\prime}-v^{\prime}\right) \Delta_{v^{\prime}}^{x^{\prime}} G\left(u, v^{\prime}, y^{\prime}\right) d v^{\prime} \\
& +\left.\int_{0}^{t} d \tau \int_{\mathbb{R}^{d-1}} q\left(y^{\prime}\right) \Delta_{\tau}^{t} \frac{\partial u_{0}(\tau, y)}{\partial y_{d}}\right|_{y_{d}=0} d y^{\prime} \int_{0}^{\infty} D_{t}\left((t-\tau)^{-1 / 2} \exp \left\{-\frac{u^{2}}{2(t-\tau)}\right\}\right) G\left(u, x^{\prime}, y^{\prime}\right) d u \\
& +\left.\int_{0}^{\infty} t^{-1 / 2} \exp \left\{-\frac{u^{2}}{2 t}\right\} d u \int_{\mathbb{R}^{d-1}} G\left(u, x^{\prime}, y^{\prime}\right) q\left(y^{\prime}\right) \frac{\partial u_{0}(\tau, y)}{\partial y_{d}}\right|_{y_{d}=0} d y^{\prime}=\sum_{i=1}^{6} M_{i} \text {. }
\end{aligned}
$$

Estimating the terms $M_{i}$ on the right-hand side of (35), using the inequalities (28), (29), and (23) as well as the finite increment formula, we obtain for $\left(t, x^{\prime}\right) \in(0, T] \times \mathbb{R}^{d-1}$ :

$$
\left|\hat{\psi}\left(t, x^{\prime}\right)\right| \leq C|| \varphi \| t^{-1 / 2}
$$

Therefore, to find the unknown densities $\hat{V}_{i}\left(t, x^{\prime}\right), i \in\{1,2,3,4\}$ of the boundary conditions (3) and the conjugation conditions (4), (5), we have obtained a system of integral equations of Volterra of second kind (22), (32).

Estimates (24), (34) and (33) hold for kernels $\hat{K}_{m}, \hat{K}_{j}^{(m)},\{m, j\} \subset\{1,2\}$, and $\hat{K}$ from these equations. Inequalities (23) and (36) are valid for the functions $\hat{\psi}_{m}, m \in\{1,2\}$, and $\hat{\psi}$. All of this 
allows us to apply to the system of integral equations (22), (32) usual method of successive approximations and, as a consequence, to obtain the unknown functions $\hat{V}_{i}\left(t, x^{\prime}\right), i \in\{1,2,3,4\}$, and the following inequalities:

$$
\left|\hat{V}_{i}\left(t, x^{\prime}\right)\right| \leq C\|\varphi\| t^{-1 / 2}, \quad i \in\{1,2,3,4\},\left(t, x^{\prime}\right) \in(0, T] \times \mathbb{R}^{d-1} .
$$

The estimates (24), (34) and (37) ensure that the single-layer potentials in (21) exist and the inequalities can be done

$$
\left|u_{m j}(t, x)\right| \leq C|| \varphi \|,\{m, j\} \subset\{1,2\},
$$

where $(t, x) \in \bar{\Omega}_{T}^{(m)}, C$ is some constant.

Hence, from the estimation (15) for $r=p=0$ it follows that the inequality (38) also holds for the function $u(t, x)=u_{m}(t, x),(t, x) \in \Omega^{(m)}, m \in\{1,2\}$ from (21). In addition, we prove that the initial condition (6) is satisfied for the function $u(t, x)$ for all $x \in \overline{\mathcal{D}}$.

Let us go back to the functions $\hat{\psi}\left(t, x^{\prime}\right)$ and $\hat{V}_{m}\left(t, x^{\prime}\right), m \in\{1,2\}$ from (32). Similarly to $[9,10,17]$, using estimates (13.2) and (13.3) from [13, Ch.IV, §13], we establish that these functions are continuously differentiable with respect to the variable $x^{\prime}$ and, in addition, their derivatives satisfy the Hölder property with respect to the spatial variable. This fact confirms our a priori assumption of the existence and continuity of the derivatives $D_{i} u\left(t, x^{\prime}, 0\right)$ and $D_{i} D_{j} u\left(t, x^{\prime}, 0\right),\{i, j\} \subset\{1, \ldots, d-1\}$, as well as the possibility of using the formula (30) in solving the equation (27). This completes the proof of the classical solvability of the problem (2)-(6). As for the uniqueness of the solution, the corresponding statement can be obtained based on the maximum principle for parabolic equations (see proof of a similar statement in $[9,10,17])$.

\section{CONSTRUCTION OF THE MARKOV PROCESS}

It follows from Theorem 1 that there exists a unique solution of $u(t, x)$ of the problem (2)(6) in the domain $(t, x) \in[0, \infty) \times \overline{\mathcal{D}}=\bar{\Omega}$, which satisfies at $t=0$ the condition $u(0, x)=\varphi(x)$, where $\varphi(x)$ is a given function from $C_{b}(\overline{\mathcal{D}})$, which is considered to be a continuous extension on $\mathbb{R}^{d}$, so that $\varphi \in C_{b}\left(\mathbb{R}^{d}\right)$. Using the solution $u(t, x)$ of the problem (2)-(6), we define an one-parameter family of linear operators $\left(T_{t}\right)_{t \geq 0}$, acting in the space $C_{b}(\overline{\mathcal{D}})$. For $t>0$ and $\varphi \in C_{b}\left(\mathbb{R}^{d}\right)$ we put $T_{t} \varphi(x)=T_{t}^{(0)} \varphi(x)+T_{t}^{(1)} \varphi(x),(t, x) \in(0, \infty) \times \overline{\mathcal{D}}$, where

$$
\begin{aligned}
& T_{t}^{(0)} \varphi(x)=u_{0}(t, x), \quad(t, x) \in(0, \infty) \times \overline{\mathcal{D}} \\
& T_{t}^{(1)} \varphi(x)=u_{m 1}(t, x)+u_{m 2}(t, x), \quad(t, x) \in(0, \infty) \times \overline{\mathcal{D}}_{m}, \quad m \in\{1,2\},
\end{aligned}
$$

$u_{0}$ and $u_{m 1}, u_{m 2}, m \in\{1,2\}$ are determined by the corresponding formulas from (21), and the densities $V_{k}, k \in\{1,2,3,4\}$, which are part of the single-layer potentials $u_{m 1}, u_{m 2}$, are the solution of the system of integral equations (22), (32), which the problem (2)-(6) boils down to. Here $T_{0}=I$, where $I$ is the identical operator.

The presence of an integral image for the operator family $\left(T_{t}\right)$ makes it easy enough to check the following properties (compare with $[9,10,17])$ :

1) if $\varphi_{n} \in C_{b}\left(\mathbb{R}^{d}\right), n \in \mathbb{N}$, sup $\sup _{n}\left\|\varphi_{n}\right\|<\infty$, and for all $x \in \mathbb{R} \lim _{n \rightarrow \infty} \varphi_{n}(x)=\varphi(x)$, where $\varphi \in C_{b}\left(\mathbb{R}^{d}\right)$, then for all $(t, x) \in \bar{\Omega}$ the relation $\lim _{n \rightarrow \infty} T_{t} \varphi_{n}(x)=T_{t} \varphi(x)$ holds;

2) for all $t \geq 0, s \geq 0$ the relation $T_{t+s}=T_{t} T_{s}$ holds, which means that the family $\left(T_{t}\right)_{t \geq 0}$ is a semigroup of operators; 
3) $T_{t} \varphi(x) \geq 0$ for all $(t, x) \in \bar{\Omega}$, if $\varphi \in C_{b}(\overline{\mathcal{D}})$ and $\varphi(x) \geq 0$;

4) operators $T_{t}$ are compression, such operators that do not increase the norm of the element.

From properties 1)-4) it follows (see, for example, [3, Ch.2, §1], [21, Ch.1]) that the family of operators $\left(T_{t}\right)_{t>0}$ corresponds to some homogeneous Feller process on $\overline{\mathcal{D}}$. If we denote its transition probability by $P(t, x, d y)$, then for $T_{t} \varphi(x)$ we have the following presentation:

$$
T_{t} \varphi(x)=\int_{\overline{\mathcal{D}}} \varphi(y) P(t, x, d y), \quad t \geq 0, x \in \overline{\mathcal{D}} .
$$

As we can see, the constructed Markov process is closely related to the heat equation (2). Therefore, it is natural to treat this process as arising as a result of bonding on the common part of the boundaries of the areas $\mathcal{D}_{i}, i \in\{1,2\}$, where conjugation conditions (4), (5) are set, two parts of the wiener process with full reflection properties at points located on the outer parts of the boundaries of these areas. In addition, as already noted, the process described here can serve as a mathematical model of the physical phenomenon of diffusion in a space with membranes.

We formulate our result as the following theorem.

Theorem 2. Suppose that the conditions of Theorem 1 hold. Then the one-parameter family of linear operators $\left(T_{t}\right)_{t \geq 0}$, determined by the solution of the problem (2)-(6), generates in the area $\overline{\mathcal{D}}$ a homogeneous Markov process such that its parts at the interior points of $\mathcal{D}_{1}$ and $\mathcal{D}_{2}$ coincide with a wiener process governed by the generalized differential operator $L=\frac{1}{2} \Delta$, and their behavior at the boundary points of these domains is determined by boundary conditions (3) and conjugation conditions (4), (5).

In conclusion, as follows from $[9,10,17]$, the constructed by us in the described way Markov process can be interpreted as a generalized diffusion in the understanding of M.I. Portenko (see $[20,21])$.

\section{REFERENCES}

[1] Anulova S.V. Diffusion processes discontinuos coefficients, degenerate diffusion, randomized drift. Dokl. Akad. Nauk 1981, 260 (5), 1036-1040.

[2] Bazalii B.V. On a model problem with second derivatives with respect to geometric variables in the boundary condition for second-order parabolic equations. Math. Notes 1998, 63 (3), 411-415. doi:10.1007/BF02317790 (translation of Mat. Zametki 1998, 63 (3), 468-472).

[3] Dynkin E.B. Markov Processes. Academic Press, New York, Springer, Berlin, 1965.

[4] Eidelman S.D. Parabolic Systems. Nauka, Moscow, 1964. (in Russian)

[5] Friedman A. Partial Differential Equations of Parabolic Type. Prentice-Hall, Englewood Cliffs, 1964.

[6] Ikeda N., Watanabe S. Stochastic differential equations anddiffusion processes. North-Holland, Amsterdam-New York, Tokyo, 1981.

[7] Ivasishen S.D. Green Matrices of Parabolic Boundary-Value Problems. Vyshcha Shkola, Kyiv, 1990. (in Russian)

[8] Konenkov A.N. On the relation between fundamental solutions of elliptic and parabolic equations. Diff. equations, 2002, 38 (2), 247-256. (in Russian)

[9] Kopytko B.I., Novosyadlo A.F. The brownian motion process with generalized diffusion matrix and drift vector. Theory Stoch. Process. 2008, 14 (30) (2), 60-70.

[10] Kopytko B.I., Novosyadlo A.F. Analytical model of a generalized diffusion process with a membrane located on a curved surface. Nauk. Visnyk of the Chernivtsi Univ. Ser. Math. 2011, 1 (1-2), 64-75. (in Ukrainian) 
[11] Kopytko B.I., Portenko M.I. The problem of pasting together two diffusion processes and classical potentials. Theory Stoch. Process. 2009, 15 (31) (2), 126-139.

[12] Kopytko B.I., Tsapovska Zh.Ya. Initial boundary-value problem with Wentzel-type conjugation condition for a parabolic equation with discontinuous coefficients. J. Math. Sci. (N.Y.) 2009, 160 (3), 283-295. doi:10.1007/s10958009-9497-9 (translation of Mat. Metody Fiz.-Mekh. Polya 2008, 51 (1), 7-16. (in Ukrainian)).

[13] Ladyzhenskaya O.A., Solonnikov V.A., Ural'tseva N.N. Linear and Quasi-Linear Equations of Parabolic Type. AMS, Providence, RI, 1968.

[14] Matiichuk M.I. Parabolic and Eliptic Boundary-Value Problems with Singularities. Prut, Chernivtsi. 2003. (in Ukrainian)

[15] Mikulevicius R. The existence of solutions of the martingale problem. Lith. Math. J. 1977, 17 (4), $149-167$.

[16] Miranda K. Equations with partial derivatives of elliptic type. Publisher of foreign literature, Moscow, 1957. (in Russian)

[17] Novosyadlo A. To the question of constructing a diffusion process that admits a generalized drift vector and a diffusion matrix. Math. Visn. NTSH. 2008, 4, 227-241. (in Ukrainian)

[18] Pilipenko A.Yu. An Inrtoduction to Stochastic Differential Equations with Reflection. Universitatsverlag, Potsdam, 2014.

[19] Pogorzelski W. Równanie całkowe i ich zastosowania. Tom IV. PWN, Warszawa, 1970.

[20] Portenko N.I. Generalized Diffusion Processes. AMS, Providence, RI, 1990 (translation of Naukova Dumka, Kyiv, 1982)

[21] Portenko M.I. Diffusion Processes in Media with Membranes. Pr. Inst. Mat. Nats. Akad. Nauk Ukr., Kyiv, 1995. (in Ukrainian)

[22] Solonnikov V.A. On boundary-value problems for linear parabolic systems of differential equations of the general type. Tr. Mat. Inst. Akad. Nauk SSSR 1965, 83, 3-162. (in Russian)

[23] Taira K. On the existence of Feller semigroups with boundary conditions. Mem. Amer. Math. Soc. 1992, 99 (475), $1-65$.

[24] Wentzel A.D. On boundary conditions fot multidimensional diffusion processes. Theory Probab. Appl. 1959, 4 (2), 164-177. doi:10.1137/1104014

[25] Zaitseva L.L. On a multidimensional Brownian motion with partly reflecting membrane on a hyperplane. Theory Stoch. Process. 1999, 5 (21), (3-4), 258-262.

[26] Zhitarashu N.V., Eidel'man S.D. Parabolic Boundary-Value Problems. Shtiintsa, Kishenev, 1992. (in Russian)

Received 01.04.2020

Revised 24.06.2020

Копитко Б.І., Новосялло А.Ф. Про одну некласичну задачу для рівняння теплопровідності та пов' $я$ зану з нею напівгрупу Феллера // Карпатські матем. публ. — 2020. — Т.12, №2. - С. 297-310.

Вивчається початково-крайова задача для рівняння теплопровідності з умовою спряження типу Вентцеля, яка не вкладається у загальну теорію параболічних початково-крайових задач і відноситься до числа умовно коректних. Ї̈̈ класичну розв' язність у просторі обмежених неперервних функцій встановлено при деяких умовах методом граничних інтегральних рівнянь. Крім того доведено, шо отриманий розв'язок $є$ напівгрупою Феллера, яка представляє в розглядуваній тут області деякий однорідний узагальнений дифузійний процес.

Ключові слова і фрази: параболічний потенціал, метод граничних інтегральних рівнянь, напівгрупа Феллера, нелокальна крайова умова. 